\title{
Efecto de la quema controlada sobre el banco de semillas de gramíneas en diferentes parches del bosque de caldén en la región semiárida central Argentina
}

\author{
Ernst, R.D. ${ }^{1 @ ;}$; Morici, E. ${ }^{1,2}$; Estelrich, H.D. ${ }^{2}$; Muiño, W.A. ${ }^{1,2}$ y Ruiz, M.A. ${ }^{1,3}$
}

'Facultad de Ciencias Exactas y Naturales. Universidad Nacional de La Pampa. Argentina.

${ }^{2}$ Facultad de Agronomía. Universidad Nacional de La Pampa. Argentina.

${ }^{3}$ INTA. EEA Anguil Ing. Agr. Guillermo Covas. La Pampa. Argentina.

\section{PalABRAS CLAVE ADICIONALES}

Propágulos.

Fuego.

Pastizales naturales.

\author{
ADDITIONAL KEYWORDS \\ Propagules. \\ Fire. \\ Grasslands.
}

\section{RESUMEN}

El banco de semillas del suelo es la principal reserva de propágulos con que cuenta una comunidad vegetal para su mantenimiento, regeneración y perpetuación. Las quemas controladas pueden producir cambios en su composición y distribución. El objetivo del presente trabajo fue evaluar y comparar el comportamiento del banco de semillas germinable de gramíneas en una región del caldenal antes y después de producida una quema controlada. Se delimitaron 5 parches de vegetación dominados por gramíneas de porte bajo (parches forrajeros), 5 parches dominados por gramíneas de porte intermedio (parches no forrajeros) y otros 5 por arbustos (parches arbustivos). A su vez mediante muestreos al azar se tomaron 5 muestras de suelo por parche, las que fueron extraídas por medio de un cilindro metálico de $7 \mathrm{~cm}$ de diámetro y $4 \mathrm{~cm}$ de profundidad, permitiendo dividir a la muestra en estratos: broza, 0-2 y $2-4 \mathrm{~cm}$ de profundidad. Los estratos se colocaron en bandejas de plástico sobre una cama de siembra esterilizada y se regaron periódicamente en invernáculo. Una vez germinadas las semillas se identificaron, contabilizaron y extrajeron las plántulas, reconociéndose para todas las profundidades 8 gramíneas. Luego de la quema Piptochaetium napostaense mostró un aumento en su germinación en 0-2 cm en los parches forrajeros y no forrajeros $(p<0,05)$. Poa ligularis disminuyó su germinación en todos los parches de vegetación $(p<0,05)$. Por su parte las gramíneas no forrajeras mostraron diferentes comportamientos. Jarava ichu disminuyó su germinación en todos los parches, mientras que Nassella tenuisima y evidenciaron un aumento en la germinación a los $0-2 \mathrm{~cm}$ en los parches forrajeros. El uso del fuego controlado produce diferentes efectos sobre el banco de semilla de gramíneas dependiendo de la temperatura alcanzada, profundidad de enterrado y de la especie en consideración.

\section{Effect of controlled burning on grass seed bank in different patches of calden forest} in the semiarid central region Argentina

\section{SUMMARY}

The seed bank is the main reserve of propagules available to a plant community for its maintenance, regeneration and perpetuation. Controlled burnings can produce changes in its composition and distribution. The objective of this work is to evaluate and compare the performance of germinable seed bank in a calden forest region before and after a controlled burning is produced. Five vegetation patches dominated by small grasses (fodder patches) were delimited as well as five patches dominated by medium size grasses (non fodder patches) and five patches dominated by shrubs (shrubby patches). At the same time, five soil samples per patch were taken by random sampling with a metallic cylinder, whose dimensions were $7 \mathrm{~cm}$ diameter and $4 \mathrm{~cm}$ height. Samples were divided into three subsamples: brushwood, 0-2 cm depth, and 2-4 cm. depth. Subsamples were placed on a sterilized seed bed in plastic trays and were periodically irrigated into a greenhouse. When seedlings emerged they were identified, counted, and extracted. Eight grass seedlings were identified for all depths. After the burning, Piptochaetium napostaense showed an increase in its ability of germination for $0-2 \mathrm{~cm}$. depth in fodder and non fodder patches $(\mathrm{p}<0.05)$. Poa ligularis decreased its ability of germination in all vegetation patches $(p<0.05)$. Non forage grasses showed different performances. Jarava ichu decreased its ability of germination in all vegetation patches, while Nassella tenuissima and $N$. trichotoma showed an increase in the ability of germination for $0-2 \mathrm{~cm}$. depth in fodder patches. The use of controlled burnings produces different effects on grass seed bank depending on the temperature reached, the burial depth and species considered.

Cronología del aríículo.

Recibido/Received: 27.3.2015

Aceptado/Accepted: 29.6.2015

On-line: 16.9.2015

Correspondencia a los autores/Contact e-mail:

gradani@speedy.com.ar

\section{INTRODUCCIÓN}

El fuego forma parte del equilibrio dinámico de los sistemas naturales pero su importancia en algunos es mayor que en otros. Su relevancia está condicionada principalmente por factores climáticos y antropogénicos (Kunst, 2003) teniendo una influencia preponderante en la modelación del paisaje (Herrera et al., 2003) 
y su efecto está restringido a la severidad del mismo. Así, las primaveras húmedas favorecen la acumulación del material vegetal en el estrato herbáceo y los veranos secos reducen los niveles de humedad de estos combustibles, por lo que el riesgo de fuegos naturales en esta región se ve favorecido por la combinación de años lluviosos con períodos veraniegos secos. Este esquema ha sido profundamente alterado por la acción del hombre a través del parcelamiento de áreas naturales y la introducción del ganado doméstico, provocando cambios en la vegetación, el suelo y el banco de semillas (Llorens y Frank, 1999; Kunst y Bravo, 2003; Dussart et al., 2011). El área del caldenal está ocupada con un $82 \%$ por pastizales naturales (Viglizzo, 2011; Estelrich y Castaldo, 2014), compuestos por gramíneas invernales las cuales forman la dieta de los bovinos (Rabotnicof et al., 2013) y donde la principal actividad ganadera que se desarrolla es la cría bovina (Roberto et al., 2008; Estelrich y Castaldo, 2014).

Actualmente el bosque de caldén se encuentra con distintos grados de degradación, existiendo mucha superficie ocupada por leñosas y material no forrajero o no palatable. Esto hace que disminuya la capacidad de carga que pueden soportar estos ambientes (Estelrich y Castaldo, 2014) dado por los bajos índices de selectividad que soportan estas especies.

La reintroducción del fuego en forma de quema controlada puede ser una alternativa de manejo, la cual permitirá un mejor aprovechamiento del pastizal natural (Rabotnicof et al., 2013). Esta puede tener diferentes finalidades: reducción del material vegetal altamente combustible para evitar futuros incendios (Nazar Anchorena, 1990; Sipowicz, 1994), eliminar la fitomasa diferida de las gramíneas no forrajeras para permitir la utilización de su rebrote como fuente de alimento (LLorens y Frank, 1999) obteniendo un mayor porcentaje de proteína bruta y digestibilidad de la materia seca (Rabotnicof et al., 2013) y crear las condiciones necesarias de luz y espacio para que el banco de semillas sea activado (Morici et al., 2009; BenechArnold et al., 2014).

La respuesta de una comunidad vegetal a la quema controlada dependerá en gran medida de su composición y estructura (Bravo et al., 2003; Estelrich et al., 2005), obedeciendo su reestablecimiento o sobrevivencia, en muchos casos, exclusivamente al banco de semillas.

El banco de semillas del suelo cumple un rol fundamental en la recuperación de áreas que sufrieron drásticos procesos de disturbios (Harper, 1977). Al mismo se lo puede definir como una agrupación de diásporas, cariopsis o semillas no germinadas que constituyen un componente importante de la dinámica vegetal y una estrategia de sobrevivencia de las especies a lo largo del tiempo (Thompson y Grime, 1979). En las zonas áridas y semiáridas se lo encuentra concentrado en los primeros centímetros del suelo y su distribución horizontal es altamente agregada (Mayor et al., 2003; Morici, 2006; Morici et al., 2009).

El banco de semillas puede ser afectado por las elevadas temperaturas y/o por los constituyentes químicos del humo y las cenizas que el fuego provoca
(Maren et al., 2010; Hepper et al., 2013). Por otra parte las posibilidades de las semillas de sobrevivir a las altas temperaturas depende del grado de penetración del calor a través del perfil del suelo, de la tolerancia de las semillas al calor y de la profundidad de autoenterrado (Auld et al., 2000; Ávila et al., 2010). El objetivo del presente trabajo fue evaluar en un área del bosque de caldén de la provincia de La Pampa, el efecto que produce la quema controlada, sobre el banco de semillas de gramíneas en distintos parches de vegetación según las distintas profundidades de enterrado.

\section{MATERIAL Y MÉTODOS}

\section{ÁREA DE ESTUDIO}

El caldenal es un sistema ecológico que se encuentra en la porción más austral de la Provincia Fitogeográfica del Espinal. Esta región ocupa una superficie de $169333 \mathrm{~km}^{2}$ equivalente al $55 \%$ de la superficie total de la región del Espinal (307374 km²) (Cabrera, 1976). En la provincia de La Pampa el bosque de caldén se ubica entre las isohietas de 350 y $580 \mathrm{~mm}$, habitando fundamentalmente en las depresiones y valles transversales, en suelo franco arenoso fino profundo. Está orientado en el Norte de la provincia de SW a NE y en el Sur se agrupan en forma de abanico orientados de E a SW (Cano et al., 1980). La temperatura media del mes más frío (julio) es $7,3^{\circ} \mathrm{C}$ y del mes más cálido (enero) $23^{\circ} \mathrm{C}$ con temperaturas extremas $\left(44^{\circ} \mathrm{C}\right.$ Máx. absoluta y $-16^{\circ} \mathrm{C}$ Mín. absoluta). El período medio libre de heladas es aproximadamente de 200 días (Casagrande y Conti, 1980).

El área de estudio se encuentra ubicada en el Establecimiento Bajo Verde (NE: Lat. 36²9'18.0" Long

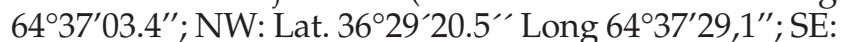
Lat. $36^{\circ} 29^{\prime} 42,4^{\prime \prime}$ Long $64^{\circ} 37^{\prime} 03,7^{\prime \prime}$; SW: Lat. $36^{\circ} 29^{\prime} 42,2^{\prime \prime}$ Long 64 $37^{\prime} 28,9^{\prime \prime}$, a $235 \mathrm{msn} / \mathrm{m}$ ), propiedad de la Universidad Nacional de La Pampa y tiene una superficie de 1980 ha.

En la actualidad, en el bosque de caldén, se puede distinguir un estrato arbóreo en el que domina Prosopis caldenia Burkart, caldén acompañado por Prosopis flexuosa DC. var flexuosa algarrobo y Geoffroea decortican (Hook. and Arn.) Burkart chañar y un estrato arbustivo, de muy variable composición florística, existiendo Condalia microphylla Cav. piquillín, Lyciun chilenses Miers llaollín y Ephedra triandra Tul. em J. H. Hunziker tramontana, entre otras. En el estrato herbáceo se pueden encontrar especies de porte bajo, generalmente con buen valor forrajero (Poa ligularis Nees ex Steud. unquillo, Piptochaetium napostaense (Speg.) Hack. flechilla negra, Nassella tenuis (Phil.) Barkworth flechilla fina, entre otras y especies de altura intermedia de poco o nulo valor forrajero (Nassella tenuisima (Trin.) Barkworth paja, Jarava ichu Ruiz and Pav. paja blanca, Amelichloa brachychaeta (Godr.) Arriaga and Barkworth pasto puna y Nassella trichotoma (Nees) Hack. ex Arechav paja. (Cano, 1988; Llorens y Frank, 1999, Morici et al., 2009).

\section{SELECCIÓN Y DESCRIPCIÓN DE LAS ÁREAS DE MUESTREO}

Dentro del establecimiento Bajo Verde el potrero seleccionado presenta una superficie de $52 \mathrm{ha}$, de donde se retiraron los animales del pastoreo una estación de 
crecimiento antes de la realización de la quema controlada, con el objetivo de acumular biomasa combustible en forma homogénea para que el fuego se pueda propagar horizontalmente sin ninguna dificultad y no dañar las copas de los árboles y arbustos.

En el mes de marzo de 2008, antes de la quema, se seleccionaron 5 áreas ubicadas en el medio del potrero, evitando así, un sector sobrepastoreado o con subutilización de forraje. En cada una de estas áreas se eligieron tres tipos de parches de aproximadamente $25 \mathrm{~m}^{2}$ cada una donde se fijaron 15 unidades experimentales (10 repeticiones por parche, donde se procedió a determinar la composición florística, en parcelas de $0,25 \mathrm{~m}^{2}$.siguiendo la metodología descripta por Cano, 1988). Estos parches (Wittemberger, 1981, citado en Barrows, 1996) fueron seleccionados teniendo en cuenta su homogeneidad en cuanto a la vegetación: parches forrajeros, no forrajeros y arbustivos. El parche forrajero presenta un estrato graminoso-herbáceo con predominio de Piptochaetium napostaense acompañado de Poa ligularis. El parche no forrajero presenta un estrato graminoso-herbáceo siendo las especies más relevantes Nassella trichotoma, N. tenuissima y Jarava ichu. Por último, el parche arbustivo posee un predominio de Prosopis caldenia, Condalia microphylla, Schinus johnstonii, con presencia en el estrato graminoso-herbáceo de $N$. trichotoma, N. tenuissima y J. ichu, acompañadas en menor medida por gramíneas forrajeras.

\section{DETERMINACIÓN DEL MATERIAL COMBUSTIBLE}

La determinación de la biomasa se realizó antes de la quema (13 de abril de 2008) por medio del método de corte y pesada (Estelrich y Cano, 1985), con 10 cortes por parche en parcelas de $0,25 \mathrm{~m}^{2}$. La composición de la biomasa aérea de vegetación o carga de combustible de los parches forrajeros y no forrajeros estuvo compuesto por material graminoso-herbáceo mientras que para los parches arbustivos además del estrato graminosoherbáceo se le incorporó el estrato arbustivo. Luego del corte manual (con tijeras), el material cosechado se llevó a laboratorio y se colocó en estufa a $60^{\circ} \mathrm{C}$ para determinar materia seca. Esta tarea se realizó dos días antes de que se efectuara la quema controlada.

\section{Medición de las temperaturas alCanzadas en El SUelO}

Antes a la realización de la quema se instalaron, en los distintos parches de vegetación, un conjunto de crayones sensibles al calor (tizas termofundibles o tempils), con la finalidad de determinar las temperaturas máximas alcanzadas en el suelo durante la quema. Estos se colocaron en sobres de aluminio y se depositaron sobre la superficie del suelo, a 2 y $4 \mathrm{~cm}$ de profundidad sin disturbar el terreno. Los crayones permitieron cubrir un rango de temperaturas desde los $52^{\circ} \mathrm{C}$ a $649^{\circ} \mathrm{C}$. Se utilizaron 10 tizas correspondientes a: $52^{\circ} \mathrm{C}, 93^{\circ} \mathrm{C}, 121^{\circ} \mathrm{C}, 163^{\circ} \mathrm{C}, 204^{\circ} \mathrm{C}, 246^{\circ} \mathrm{C}, 316^{\circ} \mathrm{C}, 427^{\circ} \mathrm{C}$, $438^{\circ} \mathrm{C}$ y $649^{\circ} \mathrm{C}$ (Sawczuk, 2009).

\section{REALIZACIÓN DE LA QUEMA CONTROLADA}

La quema controlada fue realizada el 15 de abril de 2008, por personal especializado de Defensa Civil perteneciente al Ministerio de Gobierno, Seguridad y Justicia de la provincia de La Pampa. Las condiciones climáticas durante la quema fueron: $25^{\circ} \mathrm{C}$ de tempera- tura ambiente promedio, $25 \%$ de humedad relativa del aire y vientos del NE oscilando entre 6 y $20 \mathrm{~km} / \mathrm{h}$; estas condiciones coinciden con las recomendaciones para efectuar quemas controladas realizadas por Sipowicz (1994) y Llorens (2013). La forma de realizar la quema fue inicialmente en contra del viento para finalizarla a favor del mismo (Nazar Anchorena, 1990). Se tomaron todos los recaudos para evitar su propagación hacia campos linderos.

\section{EXTRACCIÓN DE LAS MUESTRAS DE SUELO Y ANÁLISIS DEL BANCO DE SEMILLAS}

Antes de la quema (13 de abril de 2008), para cada uno de los parches, fueron extraídas 5 muestras al azar (distribución horizontal), por medio de un cilindro metálico de $7 \mathrm{~cm}$ de diámetro y $4 \mathrm{~cm}$ de altura, el cual presenta divisiones transversales. Esto permitió en el momento de la extracción de la muestra, poder dividirla en tres submuestras: broza o mantillo superficial, 0-2 $\mathrm{cm}$ y 2-4 cm (distribución vertical), haciendo un total de 225 muestras por época de muestreo. La submuestras de la broza se separa de las demás por medio de una cuchara grande. Y las restantes se dividieron introduciendo una espátula en la división transversal que presenta el cilindro. Una vez recolectadas los distintos estratos en el campo se los colocó en bolsas de plástico separadas, previamente etiquetadas y acondicionadas para su traslado a invernáculo. De esta forma cada fracción de suelo (sin subdividir) estaba compuesta por $154 \mathrm{~cm}^{3}$ totalizando así $770 \mathrm{~cm}^{3}$ de suelo en cada parche y por fecha de muestreo, coincidiendo con el volumen recomendado por Roberts (1981), con el cuál se logra detectar la mayoría de las especies presentes en el banco. Luego de la quema controlada (18 de abril de 2008) y usando la misma metodología se recogió el mismo volumen de suelo para cada parche, con la finalidad de hacer posible la comparación del banco antes y después de la quema.

Los estratos fueron llevados a laboratorio y se dejaron secar a temperatura ambiente, hasta que fue posible su tamizado, donde se les extrajo fragmentos pequeños de plantas, insectos y heces. De esta manera se redujo el tamaño de las muestras de suelo, quedando preparadas para ser puestas a germinar.

Ya en invernáculo, los estratos, fueron depositadas y esparcidas de manera uniforme en bandejas plásticas de germinación de $11 \mathrm{~cm} \times 15 \mathrm{~cm}$, las que fueron rotuladas para su identificación y perforadas previamente para permitir el drenaje del exceso de agua de riego. Cada bandeja contenía un sustrato o cama de siembra (arena fina) de aproximadamente $2 \mathrm{~cm}$ de espesor, la cual fue esterilizada, evitando que germinen semillas extrañas a la muestra. La función de este sustrato fue brindar un soporte y retener la humedad de la muestra, como también poder ofrecerle a todas las semillas allí depositadas, las mismas oportunidades de germinar y enraizar (Piudo y Cavero, 2005). De esta manera permitió que todas las muestras quedaran expuestas a iguales condiciones de luz, humedad y temperatura, de acuerdo a lo recomendado por Dalling et al. (1994) e ISTA (2013).

En el último mes de observación y extracción de plántulas las muestras dejaron de ser regadas durante 
10 días. Con este tratamiento de sequedad y calor, se buscó lograr las condiciones para romper la dormancia de la mayor cantidad posible de semillas (Acosta y Agüero, 2001; Haretche y Rodríguez, 2006; Batalla y Benech-Arnold, 2010; Benech-Arrnold et al., 2014).

Seguidamente se regaron por primera vez a capacidad de campo, con un rociador, el cual simuló una lluvia, evitando encharcamientos y desplazamientos de sedimentos. Semanalmente se irrigaron las bandejas según el requerimiento del material, y antes de mostrar síntomas de sequía, se las volvía a regar. La incubación se realizó en condiciones semicontroladas, con un período de luz de 16 horas diarias, a una temperatura aproximada de $10^{\circ} \mathrm{C}$ por la noche y de $20^{\circ} \mathrm{C}$ durante el día.

Para la determinación de la composición del banco de semillas de gramíneas, se utilizó el método de germinación directa o de emergencia de plántulas descrito por Roberts (1981) y Ernst y Morici (2013) el cual se realizó bajo condiciones controladas (Sokal y Rohlf, 1981) y se refiere al componente activo del banco o sea al aporte que tiene el banco en la brotación o germinación de sus disemínulos (Mayor, 1996; Acosta y Agüero, 2001). Este método es práctico cuando se quiere determinar el potencial de regeneración de una determinada comunidad o cuando se trabaja con ejemplares donde se conoce sus características vegetativas y sus semillas.

Según menciona Kissmann y Groth (1992) para ayudar al reconocimiento y clasificación de las semillas y plántulas de gramíneas se utilizó bibliografía disponible, la cual ayudó a analizar las estructuras vegetativas de las plántulas. También se utilizó como referencia material de herbario (Herbario de la Facultad de Agronomía, UNLPam) y cuando se dificultaba observar e identificar a la semilla o se encontraba deteriorada, se utilizó el criterio de la forma de la primera hoja que sale del coleoptile, siendo lineal y vertical para las gramíneas invernales mientras que curvadas y anchas en las estivales (Haretche y Rodríguez, 2006).

Una vez producida la germinación de las semillas, se contabilizaron las plántulas que iban emergiendo durante 8 meses, es decir desde el montaje del ensayo hasta que no se visualizó ninguna germinación de gramíneas. Una vez germinadas las semillas e identificadas las plántulas taxonómicamente, con la ayuda de una lupa binocular, pinza y aguja histológica, se eliminaron las plántulas para evitar fenómenos de competencia inter e intraespecífica (Morici et al., 2003). Este procedimiento se realizó durante los dos primeros meses en forma diaria y los últimos meses en forma semanal.

Los ejemplares dudosos que no se pudieron identificar y determinar su taxón se transplantaron en macetas, a la espera de que completen su ciclo de vida y que su clasificación fuera factible.

Mensualmente se desagregaron y removieron los sustratos de las bandejas a fin de evitar compactación y romper la costra superficial para reducir el impedimento físico de la germinación de las diásporas y emergencia de las plántulas (Acosta y Agüero, 2001; Piudo y Cavero, 2005). El tiempo total del ensayo fue

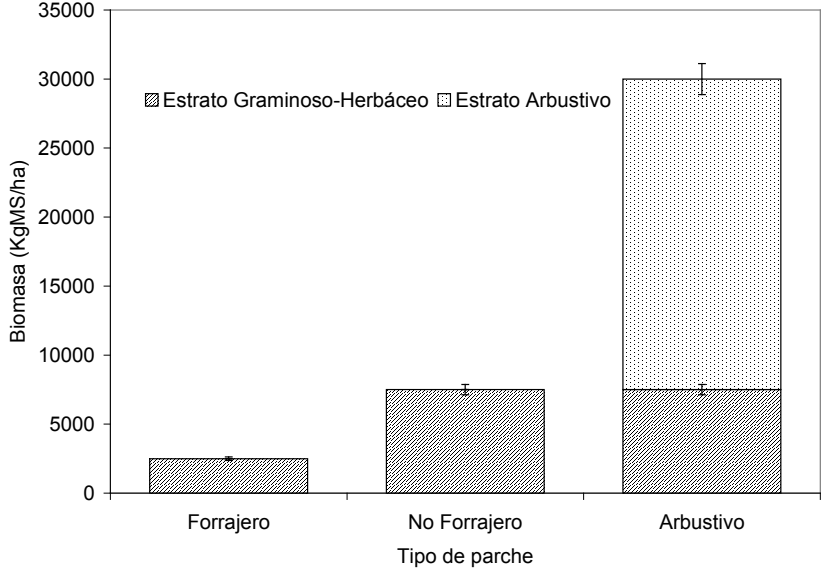

Figura 1. Biomasa aérea acumulada antes de la quema prescripta (Biomass accumulated before prescribed burning).

de 8 meses (mediados de abril hasta principios de diciembre).

\section{ANÁLISIS ESTADÍSTICO}

En cada estrato del suelo y especie en particular, las diferencias entre pre y post quema de semillas germinadas de gramíneas fueron detectadas mediante ANOVA de acuerdo a un diseño de parcelas divididas, donde la parcela principal es el parche de vegetación y la parcela secundaria es el momento de muestreo, utilizándose Tukey $(p<0,05)$ para la comparación de medias. Para el estudio del análisis estadístico de los datos se utilizó el paquete estadístico InfoStat versión 2012 (Di Renzo et al., 2012).

\section{RESULTADOS Y DISCUSIÓN}

En ambientes propensos a la ocurrencia de fuegos, que son manejados con quemas controladas para reducir los riesgos de incendios, debe tenerse en cuenta la cantidad, distribución y tipo de combustible, tanto como la relación entre temperatura y tiempo de exposición al fuego. Por otra parte se deben considerar los efectos producidos sobre las plantas y especialmente sobre el banco de semillas, ya que éste último contribuirá en gran medida en la recuperación del pastizal.

En éste trabajo, realizado en un área de bosque de caldén, la biomasa existente antes de la quema varió según el parche y estrato de vegetación analizado (figura 1). Para el estrato graminoso-herbáceo de los parches forrajeros la biomasa fue de $2496 \mathrm{~kg} \mathrm{MS}$.ha $^{-1}$ $( \pm 140)$, mientras que en los parches no forrajeros la

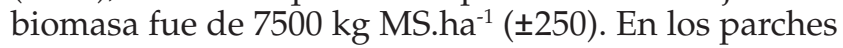
arbustivos, el combustible fue para el estrato arbustivo $22500 \mathrm{~kg}$ MS.ha $^{-1}( \pm 400)$ y la del estrato graminosoherbáceo fue de $7500 \mathrm{~kg} \mathrm{MS.ha-1}( \pm 250)$.

La cantidad de combustible existente en un área revela si el fuego se va a propagar o no, y además indica el calor que liberará la quema. Bóo et al., 1997 menciona que para ambientes semiáridos debe existir

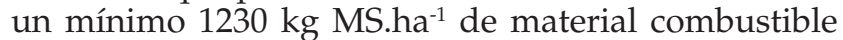
fino seco y disperso de manera homogénea para que el fuego pueda propagarse. Estos valores coinciden con resultados de biomasa registrados para el caldenal por 
Tabla I. Descripción de las especies de gramíneas halladas en el banco de semillas germinable (Description of species of grasses found in the germinable seed bank.

\begin{tabular}{llccc}
\hline Especie & Nombre vulgar & Calidad forrajera & Crecimiento & Ciclo de vida \\
\hline Bromus catharticus Vahl & Cebadilla & $\mathrm{F}$ & $\mathrm{I}$ & $\mathrm{A}$ \\
Hordeum stenostachys Godr. & Centenillo & $\mathrm{F}$ & $\mathrm{I}$ \\
Jarava ichu Ruiz and Pav. & Paja blanca & $\mathrm{NF}$ & $\mathrm{NF}$ \\
Nassella tenuissima (Trin.) Barkworth & Paja & $\mathrm{NF}$ & $\mathrm{I}$ \\
Nassella trichotoma (Nees) Hack. ex Arechav. & Pasto puna & $\mathrm{F}$ & $\mathrm{I}$ \\
Piptochaetium napostaense (Speg.) Hack. & Flechilla negra & $\mathrm{F}$ & $\mathrm{P}$ \\
Poa ligularis Nees ex Steud. & Unquillo & $\mathrm{F}$ & $\mathrm{P}$ \\
Setaria leucopila (Scribn. and Merr.) K. Schum. & Cola de zorro & $\mathrm{I}$ & $\mathrm{P}$ \\
\hline
\end{tabular}

$F=$ forrajera (fodder); NF= no forrajera (non fodder); I=invernal (winter); $E=$ estival (summer); $A=$ anual (annual); $P=$ perenne (perennial).

Sipowicz (1994). A su vez, para quemar una sabana del Chaco Kunst et al., (2003) determinaron que se necesi-

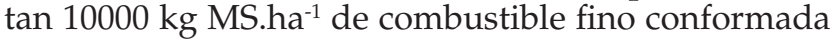
por biomasa en pie y mantillo.

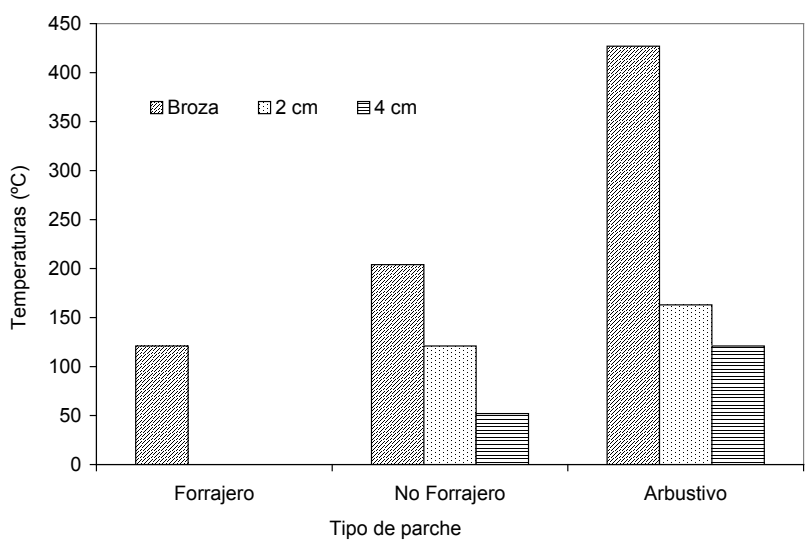

Figura 2. Temperaturas máximas registradas en el perfil del suelo bajo distintos parches de vegetación durante una quema controlada (Adaptado de Sawczuk 2009) (Maximum temperatures in the soil profile under different patches of vegetation during a controlled burn (Adapted from Sawczuk 2009)).
En las muestras de suelo colectadas antes y después de la quema fueron identificadas un total de 8 gramíneas (tabla I), dos de ellas forrajeras (P. napostaense, $P$. ligularis y 3 no forrajeras $(J$. ichu, N. tenuissima y $N$. trichotoma). Las otras tres especies también presentes en el pastizal no fueron analizadas por su baja presencia en el banco: dos forrajeras anuales invernales, Bromus catharticus Vahl var. Rupestris y Hordeum stenostachys Godr. y una forrajera perenne estival Setaria leucopila (Scribn. and Merr.) K. Schum.

Según Maestre y Cortina (2005) y Gili et al., (2010) los ecosistemas áridos y semiáridos, donde se incluye el caldenal, presentan una heterogeneidad estructural, donde la vegetación se presenta en forma no homogénea o en parches. Para los parches forrajeros la única temperatura detectada fue en la superficie del suelo y alcanzó $121^{\circ} \mathrm{C}$ (figura 2, Sawczuk, 2009). Se observó que $P$. napostaense registró un aumento en la densidad de plántulas luego de producida la quema a una profundidad de 0-2 cm con 5150 plántulas.m ${ }^{-2}$ (tabla II). $P$. ligularis tuvo una disminución en el números de diásporas germinadas en broza y los primeros centí-

Tabla II. Densidad medias de semillas germinadas (semillas.m-2) de gramíneas para los distintos parches de vegetación y profundidad de muestreo pre y post quema (Middle density of sprouts (semillas. $\mathrm{m}^{-2}$ ) of grasses for different patches of vegetation and depth of sampling pre and post burn).

\begin{tabular}{|c|c|c|c|c|c|c|c|}
\hline \multirow{2}{*}{ Especies } & \multirow{2}{*}{ Tipo de parche } & \multicolumn{2}{|c|}{ Broza } & \multicolumn{2}{|c|}{$0-2 \mathrm{~cm}$} & \multicolumn{2}{|c|}{$2-4 \mathrm{~cm}$} \\
\hline & & $A Q$ & $P Q$ & $A Q$ & $P Q$ & $\mathrm{AQ}$ & $P Q$ \\
\hline & Forrajero & $347 \pm 104^{\mathrm{a}}$ & $281 \pm 89^{a}$ & $2179 \pm 762^{b}$ & $5150 \pm 1648^{a}$ & $512 \pm 184^{a}$ & $446 \pm 133^{a}$ \\
\hline \multirow[t]{3}{*}{ Piptochaetium napostaense } & No forrajero & $67 \pm 17^{a}$ & $83 \pm 27^{a}$ & $281 \pm 81^{b}$ & $760 \pm 273^{a}$ & $132 \pm 34^{a}$ & $148 \pm 34^{a}$ \\
\hline & Arbustivo & $462 \pm 180^{a}$ & $67 \pm 16^{b}$ & $1436 \pm 502^{a}$ & $545 \pm 136^{b}$ & $215 \pm 60^{a}$ & $16 \pm 5^{\mathrm{b}}$ \\
\hline & Forrajero & $2658 \pm 926^{a}$ & $83 \pm 29^{b}$ & $1205 \pm 301^{a}$ & $132 \pm 41^{\mathrm{b}}$ & $83 \pm 24^{a}$ & $49 \pm 15^{a}$ \\
\hline \multirow[t]{3}{*}{ Poa ligularis } & No forrajero & $1205 \pm 433^{a}$ & $33 \pm 10^{b}$ & $67 \pm 20^{a}$ & $67 \pm 16^{a}$ & $33 \pm 9^{a}$ & $33 \pm 10^{a}$ \\
\hline & Arbustivo & $3054 \pm 1128^{a}$ & $33 \pm 9^{b}$ & $2988 \pm 956^{a}$ & $67 \pm 20^{b}$ & $182 \pm 60^{a}$ & $33 \pm 9^{b}$ \\
\hline & Forrajero & $83 \pm 24^{a}$ & $16 \pm 4^{\mathrm{b}}$ & $16 \pm 5^{\mathrm{a}}$ & $16 \pm 5$ & $16 \pm 4^{a}$ & $16 \pm 5^{a}$ \\
\hline \multirow[t]{3}{*}{ Jarava ichu } & No forrajero & $893 \pm 250^{a}$ & $413 \pm 115^{b}$ & $363 \pm 101^{a}$ & $116 \pm 33^{b}$ & $33 \pm 10^{a}$ & $33 \pm 10^{a}$ \\
\hline & Arbustivo & $397 \pm 121^{a}$ & $165 \pm 47^{b}$ & $99 \pm 28^{a}$ & $33 \pm 8^{b}$ & $33 \pm 9^{a}$ & $33 \pm 10^{b}$ \\
\hline & Forrajero & $198 \pm 61^{a}$ & $231 \pm 64^{a}$ & $148 \pm 42^{\mathrm{b}}$ & $314 \pm 91^{a}$ & $116 \pm 33^{a}$ & $116 \pm 35^{a}$ \\
\hline \multirow[t]{3}{*}{ Nassella tenuissima } & No forrajero & $1584 \pm 285^{a}$ & $1073 \pm 118^{b}$ & $1090 \pm 196^{a}$ & $709 \pm 106^{b}$ & $49 \pm 15^{a}$ & $49 \pm 15^{\mathrm{a}}$ \\
\hline & Arbustivo & $1122 \pm 280^{\mathrm{a}}$ & $628 \pm 157^{\mathrm{b}}$ & $1039 \pm 363^{a}$ & $446 \pm 115^{b}$ & $314 \pm 94^{a}$ & $33 \pm 10^{b}$ \\
\hline & Forrajero & $132 \pm 43^{a}$ & $132 \pm 39^{a}$ & $314 \pm 103^{b}$ & $1205 \pm 385^{a}$ & $529 \pm 174^{a}$ & $572 \pm 171^{a}$ \\
\hline \multirow[t]{2}{*}{ Nassella trichotoma } & No forrajero & $1519 \pm 501^{a}$ & $148 \pm 41^{b}$ & $893 \pm 276^{a}$ & $397 \pm 131^{b}$ & $314 \pm 94^{a}$ & $99 \pm 28^{a}$ \\
\hline & Arbustivo & $2295 \pm 826^{a}$ & $495 \pm 158^{b}$ & $2080 \pm 624^{a}$ & $462 \pm 143^{b}$ & $429 \pm 124^{a}$ & $165 \pm 47^{b}$ \\
\hline
\end{tabular}

Letras distintas indican diferencias significativas $(p \leq 0,05)$ para cada especie dentro de un mismo parche entre situaciones de prequema $(A Q)$ y postquema $(P Q)$. 
metros del suelo, luego de producida la quema. Por su parte en las gramíneas no forrajeras se observaron diferentes comportamientos. Jara ichu tuvo una disminución de plántulas en broza una vez producida la quema, mientras que $N$. tenuissima y $N$. trichotoma tuvieron sus máximos valores luego de producida la quema a la profundidad de 0-2 cm con 314 y 1205 plántulas. $\mathrm{m}^{-2}$ respectivamente. No se encontraron diferencias significativas para ninguna de las especies de gramíneas observadas para la máxima profundidad (tabla II).

Los parches dominados por especies no forrajeras (tabla II) durante la quema alcanzaron temperaturas de $204^{\circ} \mathrm{C}, 121^{\circ} \mathrm{C}$ y $52^{\circ} \mathrm{C}$ para broza, $0-2 \mathrm{~cm}$ y $2-4 \mathrm{~cm}$ respectivamente (figura 2, Sawczuk, 2009). Se observó que $P$. napostaense incrementó la densidad de plántulas a 0-2 cm, con 760 plántulas. $\mathrm{m}^{-2}$ luego del fuego. La otra gramínea forrajera, $P$. ligularis, tuvo una disminución en el número de cariopsis germinados sobre la superficie del suelo en las áreas quemadas. Las gramíneas no forrajeras, J. ichu, N. tenuissima y N. trichotoma tuvieron un comportamiento similar entre ellas, ya que en las áreas quemadas se observó una disminución de plántulas en broza y 0-2 cm. A su vez $N$. trichotoma, en la máxima profundidad, fue la única gramínea que mostró diferencias significativas entre pre y post quema, con menor densidad de plántulas luego de producida la quema.

En los parches arbustivos (tabla II) se detectaron temperaturas elevadas en las tres profundidades, en comparación con los otros dos parches: $427^{\circ} \mathrm{C}, 163^{\circ} \mathrm{C}$ y $121^{\circ} \mathrm{C}$ para broza, $0-2 \mathrm{~cm}$ y $2-4 \mathrm{~cm}$ respectivamente (figura 2, Sawczuk, 2009). Para las gramíneas forrajeras $P$. napostaense y $P$. ligularis junto a las no forrajeras $N$. tenuissima y $N$. trichotoma, se observó luego de la quema, una disminución en la densidad de plántulas emergidas para las tres profundidades muestreadas. Por su parte J. ichu tuvo una disminución significativa en la densidad de plántulas en broza y 0-2 cm luego de producida la quema.

Las temperaturas registradas en este trabajo estuvieron relacionadas con el tipo de vegetación predominante en cada parche y en función de las distintas profundidades de muestreo, variando desde $52^{\circ} \mathrm{C}$ hasta $427^{\circ} \mathrm{C}$. Estos resultados coinciden con Wright y Bailey (1982) quienes indican que la temperatura sobre la superficie del suelo aumenta en relación lineal con la biomasa de combustible acumulada y mencionan que los combustibles finos no superan los $100-200^{\circ} \mathrm{C}$ en la superficie del suelo mientras que para combustibles medianos y gruesos los valores varían desde $300-400^{\circ} \mathrm{C}$ hasta $1000^{\circ} \mathrm{C}$. En el mismo sentido, Pelaéz et al. (2001) señalan para una quema controlada al sur del caldenal temperaturas comprendidas entre 300 y $400^{\circ} \mathrm{C}$ en el estrato herbáceo, mientras que para el estrato arbustivo las temperaturas fueron superiores a $600^{\circ} \mathrm{C}$.

Distintos trabajos demuestran que en las áreas naturales lo más común es que las diásporas se encuentren desde la superficie hasta los primeros tres o cuatro centímetros de profundidad y por debajo, el tamaño del banco va disminuyendo (Harper, 1977; Mayor, 1996; Marone, 1998; Cornachione, 2008; Ernst y Morici,
2013; Martín, 2014). Esto coincide con los resultados obtenidos en este trabajo donde la mayor cantidad de diásporas encontradas antes de quemar están presentes en la broza o en los dos primeros centímetros del suelo, incluida la broza, para todos los parches de vegetación evaluados.

De una manera general, la gran cantidad de plántulas se registró en la broza y concuerda con los resultados obtenidos por Márquez et al. (2002) y Haretche y Rodríguez (2006), quienes trabajando en pastizales de Córdoba y Uruguay, encontraron que el mantillo puede actuar como un importante filtro de semillas. En el mismo sentido, Mayor et al. (2003) mencionan que en el caldenal las áreas no forrajeras y arbustivas pueden crear un ambiente propicio para la deposición y almacenamiento de semillas sobre la superficie del suelo, ya que existe una mayor deposición de sedimentos, broza u hojarasca, comparado con las áreas forrajeras. Sin embargo, trabajos realizados en banco de semilla germinable en distintos pastizales naturales de Sud América, mencionan que la mayor densidad de plántulas se detectaron en los primeros centímetros del suelo, aduciendo que en el mantillo no existen las mejores condiciones de germinar y que están más propensas al ataque por depredadores y daños causados por factores ambientales (Moscoso Marín y Díez Gómez, 2005).

La mayor densidad de plántulas después de la quema se registró para $P$. napostaense en los parches forrajeros y no forrajeros. Ello podría explicarse a partir de la profundidad de enterrado de algunas semillas, siendo una estrategia de sobrevivencia de algunas especies, como $P$. napostaense que por medio del antopodio que presenta su caripse puede anclarse en el suelo (Rúgolo de Agrazar et al., 2005; Benvenuti, 2007) y autosembrarse por presentar aristas geniculadas que actúan como palancas. Esto le permite encontrar mejores condiciones de humedad en contacto con el suelo, evitar la predación (Larrusse et al., 2012) e impedir que las altas temperaturas superficiales de un fuego afecten a las semillas. También presentan la misma estrategia $N$. tenuissima y N. trichotoma, pero sus semillas por ser más pequeñas y livianas (Rúgolo de Agrazar et al., 2005; Benvenuti, 2007) las hace mas susceptibles a los efectos del fuego. Las mayores densidades de plántulas de estas especies se registraron en los parches no forrajeros. Esto podría explicarse a partir de la estimulación de la germinación por ruptura de la dormición gracias a bajas temperaturas alcanzadas durante la quema.

Por el contrario, para P. ligularis y J. ichu, luego de la quema se registraron las menores densidades en las plántulas provenientes de la broza en todos los parches. Esto estaría indicando la sensibilidad de las semillas de estas dos especies a los efectos del fuego, ya que por sus características de menor biomasa y tamaño junto con la presencia de pelos hace que sus semillas quedan totalmente expuestas y sean destruidas (tabla II).

Varios trabajos realizados en el caldenal mencionan que las semillas de gramíneas perennes dominantes (P. napostaense, N. tenuissima y N. trichotoma) presentan dormancia (Cabeza, 1989; Mayor et al., 2003; Morici, 2006). Esta característica podría ser ventajosa para su- 
perar períodos críticos (Batalla y Benech-Arnold, 2010; Chantre, 2010; Benech-Arrnold et al., 2014). Una vez que las semillas se dispersan y penetran en el suelo, quedan a la espera de un estímulo favorable, como son las adecuadas temperaturas de un fuego, las cuales interrumpirán esa dormición para luego germinar (Rodríguez-Trejo, 2008).

Cuando las temperaturas y tiempos de exposición son más elevados los efectos sobre el banco de semillas son más negativos. Estos resultados indicarían que las elevadas temperaturas alcanzadas en los parches arbustivos disminuyeron la germinación de las cinco especies analizadas (tabla II). Esto concuerda con lo hallado para otros sistemas naturales, donde la quema redujo drásticamente la emergencia de plántulas. Esto fue observado en distintos matorrales del mundo (Pierce y Cowling, 1991; Ferrandis et al., 1999; Flores Garnica, 2009; Martínez-Orea et al., 2010), en ambientes templados (Luzuriaga et al., 2005) y en climas mediterráneos (Espósito et al., 2006; Crosti et al., 2006). Por otra parte Ávila et al. (2010), mencionan que temperaturas cercanas a los $120^{\circ} \mathrm{C}$ con tiempos de exposición de 20 minutos ya serían perjudiciales para la viabilidad de las semillas.

\section{CONCLUSIÓN}

Los efectos de la quema sobre el banco de semillas dependerán de la especie considerada, de la profundidad de enterrado y de las temperaturas alcanzadas en el perfil del suelo. De acuerdo a los resultados obtenidos en este trabajo, se observa que los fuegos controlados, realizados a fines del verano o principios del otoño, podrían favorecer aquellas especies de gramíneas forrajeras y no forrajeras que tengan la capacidad de auto enterrarse. Por otra parte, perjudicarían a las especies de gramíneas cuyas diásporas queden retenidas sobre la vegetación o la broza. Esta respuesta diferencial al fuego observada en el banco de semillas de gramíneas permitiría manipular la composición vegetal de los pastizales naturales, favoreciendo aquellas especies forrajeras que tengan la capacidad de evitar la acción de los fuegos mediante el autoenterrado. En aquellas situaciones donde las especies forrajeras posean diásporas susceptibles a los fuegos como el caso de $P$. ligularis, debería evitarse el uso de esta herramienta de manejo durante la época de producción de semillas y establecimiento de las plántulas.

\section{AGRADECIMIENTOS}

El presente trabajo fue financiado a través del Proyecto de Investigación Efectos de la Quema Controlada sobre la Vegetación y el Banco de Semilla. Proyecto No 021 perteneciente al Departamento de Recursos Naturales de la Facultad de Ciencias Exactas y Naturales. UNLPam. Llevado a cabo desde el 1 de enero de 2008 hasta el 31 de diciembre de 2013. Además el primer autor obtuvo una Beca de Postgrado para iniciar Doctorados y Maestrías titulada Efecto de la quema controlada sobre la vegetación y el banco de semilla germinable de gramineas en la región del caldenal de la provincia de la pampa, la cual fue dirigida por el Dr. Ernesto Morici desde Marzo de
2012 hasta marzo de 2014. Facultad de Ciencias Exactas y Naturales. UNLPam.

\section{BIBLIOGRAFÍA}

Acosta, L.W. and Agüero, R. 2001. El banco de propágulos de malezas en el agroecosistema: conocimiento actual y propuesta metodológica para su estudio. Agron Mesoamericana, 12: 141-151.

Auld, T.; Keith, D.A. and Bradstock, R.A. 2000. Patterns in longevity of soil seed-banks in fire-prone communities of south-eastern Australia. Aust J Bot, 48: 539-548.

Ávila, P.L.; Kin, A.G. y Morici, E. 2010. Influencia de la temperatura y el tiempo de exposición sobre la germinación y la emergencia de Piptochaetium napostaense (Speg) Hack. Universidad Nacional de La Pampa. Rev Fac Agron, 21: 5-18.

Barrows, E.M. 1996. Animal behavior desk reference. CRC Press. Boca Raton, FL. 672 pp.

Batalla, D. and Benech-Arnold, R.L. 2010. Predicting changes in dormancy level in natural seed soil Banks. Plant Mol Biol, 73: 3-13.

Benech-Arnold, R.L.; Batlla, D.; Guglielmini; A.C. y Kruk, B.C. 2014. Ecología de malezas II: la reanudación del crecimiento y el aumento del área. In: Malezas e invasoras de la Argentina. Fernández, O.A., Leguizamón, E.S. y Acciaresi, H.A. (Ed). Editorial de la Universidad Nacional del Sur y Red de Editoriales de Universidades Nacionales. Cap. Vl. pp. 139-169.

Benvenuti, S. 2007. Weed seed movement and dispersal strategies in the agricultural environment. Weed Biol Manage, 7: 141-157.

Bravo, S.; Giménez, A.M.; Kunst, C. y Moglia, G. 2003. El fuego y las plantas. In: Fuego en los sistemas argentinos. Kunst, C.; Bravo, S y Panigatti, J.L. (Ed). INTA. Santiago del Estero. Argentina. Cap. 6. pp. 61-70.

Bóo, R.M.; Peláez, D.V.; Bunting, S.; Elía, O. and Mayor, M.D. 1997. Effect of fire on woody species in central semi-arid Argentina. J Arid Environ, 35: 87-94.

Cabeza, C.E. 1989. Efecto del déficit hídrico en la germinación, emergencia y crecimiento de plántulas de algunas gramíneas nativas de Argentina, presentes en la provincia de La Pampa. Tesis Magister en Producción Vegetal. Universidad Nacional del Sur. 100 pp.

Cabrera, A. 1976. Regiones fitogeográficas argentinas. In: E.F. Ferreira Sobral. (Ed). Enciclopedia Argentina de Agricultura y Ganadería. $2^{\circ}$ Edición. Acme. Buenos Aires, Argentina. 90 pp.

Cano, E. 1988. Pastizales naturales de La Pampa. Tomo I. Convenio AACREA y Gobierno de la provincia de La Pampa, Argentina. 114 pp.

Casagrande, G. y Conti, H. 1980. Clima de la Provincia de La Pampa. In: Inventario Integrado de los recursos naturales de la Provincia de La Pampa. INTA- Provincia de La Pampa- Facultad de Agronomía, Universidad Nacional de La Pampa. 493 pp.

Chantre, G.R. 2010. Ecología del banco de semillas de Lithospermum arvence L. Modelado de la dormición y germinación. Tesis de Doctorado en Agronomía. Universidad Nacional del Sur. 131 pp.

Cornachione, M. 2008. Presencia, distribución vertical y acumulación de cariopsis viables de Nassella tenuis (Phil.) Barkworth y Jarava ichu Ruiz and Pav. en un banco de semillas de la provincia fitogeográfica del Monte, Argentina. Tesina de graduación de Ingeniería en Recursos Naturales y Medio Ambiente. Facultad de Ciencias Exactas y Naturales. Universidad Nacional de La Pampa. 32 pp.

Crosti, R.; Ladd, P.G.; Dixon, K.W. and Piotto, B. 2006. Post-fire germination: The effect of smoke on seeds of selected species from the central Mediterranean basin. Forest Ecol Manag, 221: 306-312.

Dalling, J.; Swaine, M. and Garwood, N. 1994. Effect of soil depth on seedling emergence in tropical soil seed-bank investigations. Funct Ecol, 9: 119-121

Di Rienzo, J.A.; Casanoves, F.; Balzarini, M.G.; Gonzalez. L.; Tablada, M. y Robledo, C.W. 2012. InfoStatversión 2012. Grupo InfoStat, Facultad Ciencias Agrarias. Universidad Nacional de Córdoba. Argentina. URL http://www.infostat.com.ar (16/02/2015). 
Dussart, E.G.; Chirino, C.C.; Morici, E y Peinetti, R.H. 2011. Reconstrucción del paisaje del caldenal pampeano en los últimos 250 años. Quebracho, 19: 54-65.

Ernst, R.D. y Morici, E. 2013. Banco de semillas germinable de gramíneas del caldenal, diferencias pre y post diseminación. Universidad Nacional de La Pampa. Rev Fac Agron, 22: 39-44.

Espósito, A.; Strumia, S.; Caporaso, S. and Mazzoleni, S. 2006. The effect of fire intensity on soil seed bank in Mediterranean macchia. Forest Ecol Manag, 234: 207-215.

Estelrich, H.D. y Cano, E. 1985. Disponibilidad forrajera y determinación de capacidad de carga de un bosque de Prosopis caldenia. Actas I Jornadas de Biología y || Jornadas de Geología de La Pampa. Universidad Nacional de la La Pampa. Revista Asociación Pampeana Profesionales Ciencias Naturales. Suplemento, 1: 30-35.

Estelrich, H.D.; Fernández, B.;Morici, E. y Chirino, C.C. 2005. Persistencia de los cambios provocados por los fuegos controlados en diferentes estructuras del bosque de caldén (Prosopis caldenia burk.). Universidad Nacional de La Pampa. Rev Fac Agron, 16: 25-30.

Estelrich, H.D. y Castaldo, A. 2014. Receptividad y carga ganadera en distintas micro regiones de la provincia de La Pampa (Argentina) y su relación con las precipitaciones. Semiárida. Universidad Nacional de La Pampa. Rev Fac Agron, 16: 7-19.

Ferrandis, P.; Herranz, J.M. and Martínez-Sánchez J.J. 1999. Fire impact in a maquis soil seed bank in Cabañeros Nacional Park (central Spain) J Plant Sci, 47: 17-26.

Flores Garnica, J.G. 2009. Impacto ambiental de incendios forestales. Instituto Nacional de Investigaciones Forestales, Agrícolas y Pecuarias. Mundi-Prensa. México. 325 pp.

Gili, A.A.; Trucco, R.; Niveyro, S.; Balzarini, M.; Estelrich, H.D.; Quiroga, A. and Noellemeyer, E. 2010. Soil texture and carbon dynamics in savannah vegetation patches of Central Argentina. Forest, Range and Wildland Soils, 74: 647-657.

Haretche, F. y Rodríguez, C. 2006. Banco de semillas de un pastizal uruguayo bajo diferentes condiciones de pastoreo. Ecol Austral, 16: 105-113.

Harper, J.L. 1977. Population biology of plants. Academic Press. London. pp. 33-111

Hepper, E.N.; Larroulet, M.S.; Belmonte, V. y Urioste, A.M. 2013. Propiedades edáficas de un suelo del caldenal pampeano en parches con diferente vegetación. Universidad Nacional de La Pampa. Rev Fac Agron, 23: 5-15.

Herrera, P.; Torrella, S. y Adámoli, J. 2003. Los incendios forestales como modeladores del paisaje en la región chaqueña. In: Kunst C.; Bravo, S y Panigatti, J.L. (Ed). Fuego en los sistemas argentinos. INTA. Santiago del Estero. Argentina. Cap. 13. pp. 145-155.

ISTA. 2013. International rules for seed testing. Switzerland: germination Part. 1. Agricultural and vegetable seed. Cap. 5. pp. 32-46.

Kissmann, K.G. y Groth, D. 1992. Plantas infestantes e nocivas. Tomo II. BASF (Ed). Brasileira S.A. 233 pp.

Kunst, C. 2003. Testimonios: el fuego en la historia y comentarios argentinos. In: Fuegos en los ecosistemas argentinos. Kunst, C.; Bravo S. y Panigatti, J.L. (Ed). INTA Santiago de Estero. Argentina. Cap. I. pp. 11-15.

Kunst, C.; Bravo, S.; Moscovich, F.; Herrera, J.; Godoy, J.y Vélez, S. 2003. Fecha de aplicación de fuego y diversidad de herbáceas en una sabana de Elionorus muticus (Spreng) O. Kuntze. Rev Chilena Hist Nat, 76: 105-115.

Kunst, C. y Bravo, S. 2003. Fuego, calor y temperatura. In: Kunst, C.; Bravo, S. y Panigatti, J. (Ed). Fuego en los sistemas argentinos. INTA. Santiago del Estero. Argentina. Cap. 4. pp. 31-48.

Larrusse, A.S.; Andrada, N.R. y Ocampo, E.N. 2012. Hongos asociados a semillas de poáceas naturales del semiárido, provincia de San Luis, Argentina. Rev Análisis Semilla, 6: 78-82.

Llorens E.M.y Frank, E.O. 1999. Aspectos ecológicos del estrato herbáceo del caldenal y estrategias para su manejo. AACREA, Subsecretaria de Asuntos Agrarios-Provincia de La Pampa, E.E.A. INTA Anguil. 81 pp.
Llorens, E.M. 2013. Caracterización y manejo de los pastizales del centro de La Pampa. Ministerio de la Producción. (Ed). Gobierno de La Pampa. 52 pp.

Luzuriaga, A.L.; Escudero, A.; Olano, J.M and Liodi, J. 2005. Regenerative role of seed banks following an intense soil disturbance. Acta Oecol, 26: 141-151.

Maestre, F.T. and Cortina, J. 2005. Remnant shrubs in Mediterranean semiarid steppes, effects of shrub size, abiotic factors and species identity on understorey richness and occurrence. Acta Oecol, 27: 161-169.

Marem, I.E.; Janovsky, Z.K.; Spindelböck, J.P.; Daws, M.I,; Kaland, P.E. and Vandvik, V. 2010. Prescribed burning of northern heathlands: Calluna vulgaris germination cues and seed-bank dynamics. Plant Ecol, 207: 245-256.

Marone, L.; Rossi, B.E, and Horno, M.E. 1998. Timing and spatial patterning of seed dispersal and redistribution in a South American warm desert. Plant Ecol, 137: 143-150.

Márquez, S.; Funes, G.; Cabido, M. y Pucheta, E. 2002. Efectos del pastoreo sobre el banco de semillas germinable y la vegetación establecida en pastizales de montaña del centro de Argentina. Rev Chilena Hist Nat, 75: 327-337.

Martín, M. 2014. El banco de semillas de gramíneas a diferentes presiones de pastoreo en relación con la distancia a la aguada, en un pastizal de la provincia de La Pampa, Argentina. Tesina de graduación de Ingeniería en Recursos Naturales y Medio Ambiente. Facultad de Ciencias Exactas y Naturales. Universidad Nacional de La Pampa. 43 pp.

Martínez-Orea, Y.; Castillo-Agüero, S.; Chávez, M.P. and Sánches, I. 2010. Post-fire seed bank in a xerophytic shrubland. Bol Soc Bot Méx, 86: 11-21.

Mayor, M.D. 1996. Banco de semillas de un pastizal-arbustal natural del sudeste de La Pampa, su variación estacional y la relación con la vegetación existente. Tesis Magister en Producción Vegetal. Universidad Nacional del Sur. 128 pp.

Mayor, M.D.; Bóo, R.M.; Peláez, D.V. and Elía, O.R. 2003. Seasonal variation of the soil seed bank of grasses in central Argentina as related to grazing and shurb cover. J Arid Environ, 53: 467-477.

Morici, E.; Ernst, R.D.; Kin, A.G.; Estelrich, H.D.; Mazzola, M.B. y Poey, M.S. 2003. Efecto del pastoreo en un pastizal semiárido de Argentina según la distancia a la aguada. Arch Zootec, 52: 59-66.

Morici, E. 2006. Efecto de la estructura del pastizal sobre el banco de semillas de gramíneas en el bosque de caldén (Prosopis caldenia) de la provincia de La Pampa (Argentina). Tesis Doctoral en ganadería Ecológica. Universidad de Córdoba. España. 143 pp.

Morici, E.; Doménech García, V.; Gómez Castro, G.; Kin, A.G.; Saenz, A.M. y Rabotnikof, C.M. 2009. Diferencias estructurales entre parches de pastizal del caldenal y su influencia sobre el banco de semillas, en la provincia de La Pampa, Argentina. Agrociencia. 43: 529-537.

Moscoso Marín, L.B. y Díez Gómez, M.C. 2005. Banco de semillas en un bosque de roble de la Cordillera central Colombiana. Rev Facultad Nacional Agr Colombia, 58: 2931-2943.

Nazar Anchorena, J.B. 1990. Fuegos controlados, parámetros para determinar la conveniencia del uso de fuegos controlados sobre las pasturas naturales. Universidad Nacional de La Pampa. Rev Fac Agron, 5: 127-129.

Peláez, D.V.; Bóo, R.M.; Mayor, M.D. and Elía, O.R. 2001. Effect of fire on perennial grass species in central semiarid. J Range Manag, 54: 617-621.

Pierce, M.S. and Cowling, M.R. 1991. Dynamics of soil-stored seed banks of six shrubs in fire-prone dune fynbos. J Ecol, 79: 731-747.

Piudo, M.J. y Cavero, R.Y. 2005. Banco de semillas: comparación de metodologías de extracción, de densidad y de profundidad de muestreo. Publicaciones de Biología. Universidad de Navarra. Serie Botánica, 16: 71-85.

Rabotnikof, C.M.; Sáenz, A.M.; Morici, E. y Lentz, B.C. 2013. Efecto de la quema invernal sobre el valor nutritivo de especies codominantes del pastizal mixto del caldenal en la región semiárida central de Argentina. Universidad Nacional de La Pampa. Rev Fac Agron, 22: 67-72. 
Roberto, Z.; Frasier, E.; Goyeneche, P.; González, F. y Ádema, E. 2008. Evolución de la carga animal en la provincia de la Pampa. Publicación Técnica $N^{\circ}$ 74. INTA EEA Anguil. La Pampa. 55 pp.

Roberts, H.A. 1981. Seed banks in soils. Adv Appl Biol, 6: 1-55.

Rodríguez-Trejo, D.A. 2008. Fire regimes, fire ecology, and fire management in Mexico. Ambio, 37: 548-556.

Rúgolo de Agrazar, Z.E.; Steibel, P.E. y Troiani, H.O. 2005. Manual ilustrado de las gramíneas de la provincia de La Pampa. Universidad Nacional de la Pampa y Universidad Nacional de Río Cuarto. Córdoba. 359 pp.

Sawczuk, N. 2009. Evaluación del efecto del tiempo de permanencia de las temperaturas máximas de quema sobre propiedades físicas y químicas de un Haplustol éntico de la región central del caldenal. Tesina de graduación de Ingeniería en Recursos Naturales y Medio
Ambiente. Facultad de Ciencias Exactas y Naturales. Universidad Nacional de La Pampa. 23 pp.

Sipowicz, A.H. 1994. Ecología y manejo del fuego en el ecosistema del caldenal. Boletín de Divulgación N 51. INTA EEA Anguil. La Pampa. 17 pp.

Sokal, R.R. and Rohlf, F.J. 1981. Biometry. The principles and practice of statistics in biological research. WH Freemna y Company. New York. 859 pp.

Thompson, K. and Grime, J.P. 1979. Seasonal variation in seed banks of herbaceous species in ten contrasting habitats. J Ecol, 67: 893-921.

Viglizzo, E.F. 2011. El agro, el clima y el agua en La Pampa semiárida: Revisando paradigmas. Acad Nac Agr Vet, 64: 251-267.

Wright, H.A. and Bailey, A.W. 1982. Fire ecology, United States and southern Canada. John Wiley and Sons. New York. 501 pp. 
